\section{Das Mauerblümchen}

\author{
E. Taverna
}

Durch die blauen Tapeten sickern die Hörner und Trompeten der übenden Adventsbläser, und durch die schalldichten Fenster braust der weihnachtliche Verkehr. Die Sitzungszimmer im «au premier» des Bahnhofs sind alle besetzt. Der Kellner macht sich auf die vergebliche Suche nach Brot.

Die junge Redaktorin ist eigens aus den Ferien hergereist, um der älteren Männerrunde das Konzept des "offiziellen PatientInnenmagazins der Ärztinnen und Ärzte» zu erläutern. Es ist nicht völlig neu, was da auf die vergangenen 30 Jahre aufbaut. Es ist ein Versuch, mit modernen Mitteln und frischen Ideen das Statut der "Sprechstunde» umzusetzen. Gemäss Vertragsentwurf des Verbandes Deutschschweizer Ärztegesellschaften VEDAG mit dem Verlag soll das viermal jährlich erscheinende Magazin «dem Laien in allgemein verständlicher Form medizinische Erkenntnisse vor allem aus den Bereichen der allgemeinen Hygiene, der Gesundheitsberatung und der Krankheitsprophylaxe vermitteln. Zugleich soll die Zeitschrift beim Leser Interesse für die Aufgaben der Angehörigen der medizinischen Berufe, im besonderen der Schweizer Ärzte und ihrer Hilfspersonen, wecken und das Verständnis für deren Anliegen fördern. Illustration sowie Beiträge und Berichte aus anderen Sachgebieten sollen die Thematik auflockern."

Das Feld soll nicht allein dem "Kassensturz" überlassen werden, der Inhalt nicht zur VerbandsPR-Masche verkommen, glaubwürdig bleiben, vermitteln und Vertrauen schaffen. Die Strategie setzt auf Dialog, auf stilistische Mittel wie offene Briefe, Meinungstexte, Tagebücher und Erlebnisberichte. Ein erster Teil, im letzten Heft bereits umgesetzt, setzt ein Schwerpunktthema, ein zweiter Teil bietet Raum für allgemeine und gesundheitsmedizinische Aktualitäten.
Die ausgebildete Journalistin definiert das gute Schreiben als Balanceakt verschiedener Stilwerte, gewichtet Verständlichkeit, Authentizität, Schönheit, Kürze und gutes Deutsch. Sie überzeugt durch ihr Engagement und ihre Ausdauer, die sich auch darin zeigt, wie sie die Passivität ihrer Auftraggeber gelassen hinnimmt. Das Layout von 4/99 überzeugt, Text und Gestaltung sind professionell, ansprechend und wohltuend sachlich im Vergleich zur Konkurrenz. Dennoch, das Unternehmen dümpelt durch die Jahrzehnte, ständig in Frage gestellt vom drohenden Austritt irgendeiner kantonalen Ärztegesellschaft, nie gestorben und doch von mässigem Erfolg. Wo die Inserate nur etwa einen Viertel der Kosten abdecken, ist jedes FMH-Vollmitglied mit seinem 55-FrankenBeitrag unentbehrlich. Ein Fünfliber bleibt dem Vorstand für seine Deutschschweizer Präsidentenkonferenzen und seine hochgesteckten Ziele im statutarischen Multipack.

Das gedruckte Produkt ist solide gemacht. Für den Beitrag gibt es fünf Nummern für das eigene Wartezimmer. Etwa 1000 der 7000 Gesellschaftsmitglieder zahlen zusätzliche 18 Franken für ein Jahresabonnement. Der neue Vertrag entlastet den Verband und überlässt dem Verlag "Rosenfluh Publikationen» Werbung, Inkasso und weitere Dienstleistungen. Die anwesende Redaktionskommission übernimmt eine Scharnierfunktion, garantiert ideelle und juristische Unterstützung und will bei Lesern und Ärzten die Akzeptanz und Verbreitung fördern. Dass selbst die betroffenen Anbieter im Markt ihr Eigenprodukt oft schlecht kennen, ist eine Tatsache.

Für das neue Jahr sind folgende vier Themen vorgesehen: Erinnern - Strahlen - Sexualität - Tiere in der Medizin. Vielleicht müsste eine Konferenz der kantonalen Präsidenten einen Arbeitstag ihrem selbstgezogenen bekannten/unbekannten Mauerblümchen widmen. Sie sind nicht die einzigen im Mediendschungel. Sie sind weder Medienprofis noch haben sie eine einheitliche Meinung. Da wird auch eine kompetente Solistin nicht genügen, denn die anderen Gärtner haben klarere Ziele und weit mehr Mittel. 